\title{
Editorial
}

\section{Stages in the Pathophysiology and Eventual Treatment of Brain Disorders}

Staging in neuropsychiatry has been adapted and, to some extent, modified from applications in oncology. The most recent Mojacar Meeting "Staging Neuropsychiatric Disorders: Implications for Etiopathogenesis and Treatment" presented the notion of staging in a forum defined by Strategies for Studying Brain Disorders. McGorry addressed the enduring issue of Diagnostic utility (cf. Palomo et al., 2008) by posing the question, is clinical staging the answer? It seems likely that staging offers a useful strategy to select safe and effective treatments, providing greater refinement, that treatments differ according to stage, as e.g. in cancer, and is more and effective in the early stages. The clinical staging model offers a continuum over disorder liability ranging from increased risk for psychosis or severe mood changes that are symptom-like but currently asymptomatic, at a " 0 " score, through scores " $1 \mathrm{a}$ ", " $1 \mathrm{~b}$ ", " 2 ", "3a", "3b", " $3 c$ ", and "4", the severe persistent unremitting illness, with indicative neurobiological and endophenotypical markers, e.g. that hippocampal volume decreases with severity in affective disorders, etc.

The question: "why go beyond genes?" was posed by Sokoloff in relation to premorbid, prodromal, onset/determining, and residual/stable stages of brain disorder, based upon the interactive influences of gene-environment, whereby "susceptibility" may be sought through epigenetics. Genomic imprinting, a widespread epigenetic phenomenon in mammals with imprinted genes expressed in the developing hypothalamus and placenta, ensures the necessary interactions through which survival, under variations in circumstance, is assured. Imprinted genes, all involved in placental development and many expressed in brain development, are expressed according to the parent by which one is "shut-down" and the other is "expressed" are adaptive in generation. Keverne reported that mutations to imprinted genes (Peg3, Peg1) influenced development of hypothalamic and functionally-related limbic structures with major interactive investments co-ordinating mother-infant physiological homeostasis to ensure mother care and nurturing. Massart et al. identified GPR88, a protein abundant in rat and primate striatum, as an orphan G protein-coupled receptor (GPCR) specific to the striatum, intimately involved in implicit memory, movement and motivation. Their findings described, hitherto unsuspected, epigenetic functions of GPR88 in differentiating cortical neurons implying distinct folding, mode of action and stabilization of this chromatin-associated GPCR. Cortical GPR88, an intranuclear protein and epigenetic factor, is expressed in the nuclei of neurons and interneurons with a role in developmental nuclear transportation.

Staging through the life span may be the periodicity in the course of the disorder, or, a particular step, or position, in the developmental process to determine the extent or progression 
of the disease state. For present purposes, the period of adolescence and adolescent vulnerability was discussed from a perspective of normative brain and behaviour development. Moghaddam et al. have chosen adolescence as a developmental period to study the extent of alterations to brain regions, linked to emotion and cognition, that underlie risks for neuropsychiatric conditions, as modeled in laboratory rats at adolescent age (28-42 days) and adult age ( $>60$ days). They indicated that adolescent rats were more susceptible to the functional activation evoked by internal and external motivating factors, e.g. hunger, or, cue presence on perseveration during the extinction of a previously-rewarded operant behaviour. By examining the influence of DA-modulated prefrontal cortical circuits, also in adolescent (4550 days-of-age) and young adult rats, using electrophysiological and behavioural analyses, O'Donnell et al. described DA-controlled fast-spiking GABA interneuron changes and switching, inhibitory-excitatory, in response to DA D2 agonists. Neonatal ventral hippocampus lesioned (NVHL) rats, an established neurodevelopmental model of schizophrenia, displayed disinhibition in the PFC during a DA-dependent decision-making task, implicating impaired cognitive functioning due to an abnormal interneuron maturation in the adult rats. Luna made the case that adolescence presented a unique period of individual development wherein numerous avenues of vulnerability, based upon predisposing characteristics, to situations that precipitate the emergence of psychopathology, e.g. schizophrenia, mood disorder and suicide are to be considered. She described several studies aimed at defining the specific systems, that underly cognitive control in adolescence, e.g. executive functioning, that characterize brain processes in development, allowing for the system to override impulsive behaviour, make definitions of culpability and mature through adolescence. Arango et al. outlined aspects of adolescent psychosis with child and adolescent debut in first episodes of the disorder, according to the "developmental stages in mental disorders", as outlined first by Kraepelin. Accordingly, the progressive loss of cortical grey matter, not only in patients with childhood-onset schizophrenia, but also in other early-onset psychoses displaying progressive brain changes that could provide predictors of diagnosis and/or prognosis in the populations under study. These patients showed deficits in grey matter volume, an excess of cerebrospinal fluid in the frontal lobe and excessive losses of grey matter in the parietal lobes. Belger recounted the possible features determining the etiopathophysiology of autism from a neurodevelopmental perspective that incorporated three domains involving language, social behaviour and repetitive restrictive behaviours. She discussed a Broad Autism Phenotype (BAP) involving, tentatively, a rigid personality, an "aloof" personality, language pragmatism and deficits in social cognition. The neural circuitry associated with the social cognitive deficits in autism included: the dorsal executive control system, the ventral social-affective processing system and the fusiform-amygdala-orbitofrontal affect system. Vanderschuren outlined a structural and neuropharmacological approach to understanding the social play behaviour of rats by examining the neural substrates of "play" behaviour, ostensible preparations for sex and aggression in the mature animal, in adolescent and adult rats. The findings that he presented indicated that interacting opioid -cannabinoid- dopaminergic systems within the corticolimbic neural circuits that were associated with incentive motivation and reward processes exerted modulatory influences upon the various expressions of social play behaviour. Laviola presented several animal models, chiefly involving the damaging role of stress, that constituted "critical age windows", wherein the disruptions of normal brain development affected the early stages of ontogeny with a pathologically enhanced sensitivi- 
ty to environmental events and an alteration of developmental processes towards less suitable directions, e.g. prenatal stress, affecting the HPA-axis with eventual predispositions for mood disorders, etc. According to the "double-hit" hypothesis, mutant gene-based deviations and specific environmental events, i.e. traumatic stress during cerebral development stages, will predict the neurobehavioural disturbances expressed in the adult individual. In young, adolescent and adult rats BDNF, necessary for both brain development and plasticity was detrimentally affected by perinatal stress. McGuire discussed the "At Risk Mental State (so-called ARMS)" studies with regard to brain imaging analyses in early stages of psychosis, the onset of psychosis that is preceded by a prodromal phase, followed by first-episode psychosis and chronic psychosis. Studies based on ARMS implicated alterations of grey matter volume, neurocognitive functioning, including the aberrant attribution of salience, as well as alteration in dopaminergic-glutamatergic balance, and reductions in thalamic glutamate. Pukrop described too the risk situation of clinically defined subjects within ARMS, that were identified by the Cologne Early Recognition and Intervention Center, with regard to genetic, epidemiologic and clinical high-risk studies on first episode and multiple episode patients. He related cross-sectional and longitudinal studies that included sample heterogeneity and ultra high-risk criteria in attentional psychological squeezing, transient psychological squeezing and reduced social/cognitive expression and functioning as basic symptoms in early and late high-risk.

Murray discussed some implications of dopamine-sensitization on the likelihood of individuals with schizophrenia-like psychoses expressing greater risk for drug-use. Much of this information was based on: (i) the general increase in consumption of cannabis over the last quarter-of-a-century, and, (ii) the enhanced potency of its street (sub-cultural) preparations available globally. In this regard, both Beninger and Abi-Dargham maintained the dopamine theme in serious psychiatric disorders. Vazquez-Barquero et al. reported the efficacy of neuroleptic treatment and relapse rate in schizophrenic first episode patients with operational definitions of Response, Remission and Recovery and the predictors of good Response and Recovery as exemplified in the Discontinuation studies (PAFIP design), that report the longterm outcome of patients undergoing the "guided discontinuation" of antipsychotic treatment. The main outcome measure was clinical relapse, as well as clinical and social predictors of relapse. High rates of relapse, after discontinuation, were demonstrated $(58.7 \%$ in the first 18 months), with risk of relapse significantly higher for patients diagnosed schizophrenia or schizophreniform disorder $(66.7 \%)$. Severity of symptoms, $1^{\text {st }}$ episode-need for hospitalization and substance abuse conferred a higher risk for relapse than the Early Signs Scale. Acuña et al. presented the contention that, underlying the expressions of self-awareness and executive functioning impaired in schizophrenia, the singular lack of insight, reductions in cognitive flexibility and the "updating of memories", working memory deficits and problems in decision-making are integral to clinical psychosis. These functional considerations implied involvement of prefrontal-subcortical circuits whereby a ventral "stream", ventral premotor cortex with working-memory, decision-making and encoding outcomes, and a dorsal "stream", dorsal premotor cortex with transformative outcomes of visual guided action, were applied to tackle the major issues of How? and Where? Lobo et al. described a strategy designed to test certain heuristic interests in "staging", namely the hypothesis that stages of cognitive impairment, e.g. mild-moderate-severe, documented with proven, unsophisticated methodology, are valid and sufficient to predict negative outcomes of dementia in particular 
and mortality in general. One pertinent question was whether or not cognitive disturbance offered an index of "biological value". They concluded that the validity of classifying stages of cognitive difficulty in elderly populations was assured: cognitive impairments were associated directly with elevated risk for dementia and also linked to enhanced risk for mortality. Wolf, Grace and Kalivas discussed the central role of glutamatergic transmission in addictive disorders. In uniting many notions around staging, Post emphasized the role of early stress, sometimes compensatory otherwise not so, influencing later stress with regard to (i) heredity, (ii) stressor, and (iii) episodic aspects of disorder. Thus, together with genetic vulnerability, multiple environmental events convey liability for illness progression, and thereby disease staging, through: 1) distal and proximal stressors, 2) recurrence, and possible exacerbation, of episodes of disorder, and 3) presence of comorbidity in the abuse of substances, e.g. cocaine. BDNF, involved in many elements of onset and treatment, in specific brain regions is reduced by repeated stressors and other circumstances detrimental to brain function and plasticity. A reconceptualization of existing notions of sensitization/cross-sensitization among stressors, episodes and affective states as severe, potentially progressive, and lethal biomedical threatens to brain and body survival with life-long monitoring and treatment was postulated.

\section{References}

Palomo T, Beninger RJ, Kostrzewa RM, Archer T. Beyond Neuropsychiatric Diagnostics: Symptoms not disorders. Madrid: Fundacion Cerebro y Mente; 2009.

Trevor Archer

University of Gothenburg

SWEDEN 